\title{
ODFF: Opposition and Dimension based Firefly for Optimal Reactive Power Dispatch
}

\author{
Brajula W \\ Department of Embedded System, \\ Udaya school of engineering \\ Kanyakumari,Tamil Nadu, India \\ w.brajula@gmail.com
}

\author{
Bibin Prasad M \\ Department of Applied Electronics, \\ Regional Centre of Anna University \\ Tirunelveli, Tamil Nadu, India \\ bibinprasad..m@gmail.com
}

\begin{abstract}
In Optimal Reactive Power Dispatch (ORPD) system, the main objective arises from the reduction of active power loss and the voltage deviation. In this paper, Opposition and Dimension based Firefly (ODFF) is proposed to achieve the objective. The experimentation is carried out in IEEE 14 and IEEE 39 benchmark bus system. The result obtained from the proposed OFDA is compared with the conventional methods like Genetic Algorithm (GA), Particle Swarm Optimization (PSO), Firefly (FF) and Artificial Bee Colony (ABC). Further, the comparative result proved that the proposed ODFF method provides less active power loss and voltage deviation with high convergence speed than those achieved by the existing methods.
\end{abstract}

Keywords: OFDM, Active power loss, Voltage deviation, ODFF

\section{Introduction}

The process of generation, transmission and distribution of power in the electrical power system is a complex task. The less use of power resources are made to withstand the security and reliability of the system [2]. Recently, (ORPD) has become as the continually increasing attention over developments of the power system. ORPD is one of the non- linear problems that should attain the minimum active power loss and voltage stability and maximum voltage deviation [25] [26]. Hence, the system is mainly controlled by the continuous and discrete control variables which include the generator bus voltages, transformer tap settings and reactive power of shunt VAR compensator. Those variables are controlled to satisfy both the equality and in equality constraints [27] [28] [29] [30].

Various classical optimization techniques such as linear programming [10], non- linear programming [8], quadratic programming [13], decomposition approach [12], diverse- integer programming [7], Newton-based method [9], dynamic programming [11] etc were used earlier to solve the ORPD problems. Since numerous local optima are present in ORPD, these aforesaid techniques are not possible to examine the global optima of the system. Further, in recent time, several meta-heuristic algorithms were implemented to overcome the limitations arising under the classical optimization methods. Those methods includes Bacterial Foraging algorithm (BFA) [15], Adaptive Genetic algorithm (AGA) [14], Particle Swarm Optimization (PSO) [16], Quantum- inspired Evolutionary algorithm (QFA) [17], Hybrid PSO (HPSO) [18], Evolutionary Programming (EP) [19] etc.

Later other advanced algorithms such as Differential Evolution (DE) [24], Chaotic Parallel Vector Evaluated Interactive Honey Bee Mating Optimization (CPVEIHBMO) [22], Enhance Firefly [20], Quasi- Oppositional Differential Evolution (QODE) [21], Hybrid Fuzzy Multi- Objective EA [23] etc were implemented by several researchers to solve the problems of the ORPD. But still the challenges such as computational complexity, non-linear and no- convex problems exist in ORPD system. 


\section{Literature Review}

\subsection{Related Works}

In 2016, Brett and Alejandro [1] have proposed the branch flow modelling approach to maintain the reactive power in the distributed energy resources of the ORPD system. They have started the implementation in the ORPD system with balanced condition. The optimal power flow (OPF) was considered as the main problem, and have used the convex quadratic programming (QP) to maintain the operating condition of the system. The computation of QP was solved by the distribution algorithm that is based on the Alternating Direction Method of Multipliers (ADMM). Further, they have used the same procedure to solve the OPF problem of ORPD system under unbalanced condition.

In 2016, Seyed et al. [3] have brought about the stochastic multi- objective ORPD (SMO-ORPD) approach to control the ORPD problems in the wind integrated power system. They have considered the main objectives such as reduction of active power loss and voltage stability. The combination of constraint method and fuzzy satisfying approach were used to solve the proposed optimization. The implementation was done using IEEE-57 bus system and those were performed under the GAMS environment. The ability of the proposed method to deal with the minimization of active power loss and voltage stability was proved precisely by comparing the proposed method with the conventional intelligent search- based algorithms.

In 2015, Aparajita Mukherjee and Vivekananda Mukherjee [4] have suggested the chaotic krill herd algorithm (CKHA) which is one of the evolutionary- based techniques to control the problems concerning the ORPD. Here the variables such as voltages of bus, transformer tap positions and reactive power sources were measured for the experimentation. They have implemented the algorithm to minimize the active power loss and voltage deviation and improving the voltage stability index. The efficiency and convergence speed of the algorithm was achieved by comparing the proposed method with the existing algorithms.

In 2016, M. Basu [5] has adopted the multi- objective differential evolution (MODE) in order to solve the problems under the ORPD system. Those problems were solved by reducing the voltage deviation and active power loss and enhancing the voltage stability. They have varied the control variables such as transformer tap settings, terminal voltages of the transformer, reactive power output of shunt VAR compensators. The experimentation was carried out in IEEE 30-bus, 57-bus and 118-test bus systems. The superior dispatch decisions were acquired by the proposed algorithm when compared with strength pareto evolutionary algorithm 2 (SPEA 2 ) on the root of pareto-optimal solutions.

In 2015, Mohd Herwan et al. [6] have developed the gray wolf optimizer (GWO) which is one of the new meta-heuristic techniques done to solve the issue arising from the ORPD. The group of control variables such as ratio of transformer taps settings and the generator voltages were varied to perform the experimentation. Those variables were varied to attain the minimized power loss and voltage deviation, and the corresponding experiment was prepared in IEEE 30 and IEEE 118- test bus system. The supremacy of the GWO algorithm was achieved when compared with other algorithms.

\subsection{Review}

The review stated the operation of different algorithms applied to solve the problem of ORPD. A number of recent methods such as ADMM [1], SMO-ORPD [3], CKHA [4], MODE [5], and GWO [6] were effectively reported in the literature. However some problems stay as the main challenges that affect the operation of ORPD. General challenges under ORPD includes the slow regulation corresponds to control voltage deviation, problems regarding the uncertain parameters, scheduling of hydro thermal problems, geometric optimization problem, derivative control etc. Therefore, ORPD system requires an improved method to handle those challenges in an efficient manner.

\section{Model of ORPD}

The general objective of ORPD is the minimization of active power loss and voltage deviation and the maximization of voltage stability index which is represented in eq. (1) where A denotes active power loss and $\mathrm{B}$ denotes deviation of voltage.

$$
O_{b}=a A+(1 \quad a) B
$$

The selected variables are responsible for creating the in equality and equality constraints. The vectors of the dependant variables are expressed in eq. (2) where $P d_{G}$ represents the slack bus power, 
$\mathrm{Vd}_{\mathrm{lj}}$ indicates the voltage of bus $\mathrm{PQ} j=1,2 \ldots . N P Q, \mathrm{Qd}_{\mathrm{Gj}}$ denotes the reactive power output of the generator $\mathrm{j}=1,2 \ldots . \mathrm{NG}, \mathrm{NG}$ and NPQ is the count of generator bus and PQ buses.

$$
X=\left[P d_{G 1}, V d_{l}{ }_{1} \ldots \ldots . . V d_{l} \quad N P Q, Q d_{G}{ }_{1} \ldots \ldots . Q d_{G} \quad N G\right]
$$

The vector of control variables are expressed in eq. (3) where $\mathrm{Vc}_{\mathrm{Gj}}$ denotes the terminal voltage of the voltage controlled bus, $j=1,2 \ldots . N G, Q c_{C j}$ indicates the output of the shunt VAR compensator $j=1,2 \ldots . N C, \mathrm{Tc}_{\mathrm{i}}$ denotes the tap setting of the tap changing transformer $j=1,2 \ldots . N G, \mathrm{NC}$ and NT are the count of shunt VAR compensators and the tap settings transformers.

$$
U=\left[V c_{G}{ }_{1}, \ldots . . V c_{G}{ }_{N G}, Q c_{C}{ }_{1} \ldots . . Q c_{C}{ }_{N C}, T c_{1}, \ldots . . T c_{N T}\right]
$$

Minimization of active power loss: The formulation for the minimization of active power loss is represented in eq. (4) where $\mathrm{g}_{\mathrm{i}}$ is the $\mathrm{i}^{\text {th }}$ division conductance between $\mathrm{k}^{\text {th }}$ and $1^{\text {th }}$ buses, $\delta_{\mathrm{k}}$ and $\delta_{1}$ are the voltage phase angles of the $\mathrm{k}^{\text {th }}$ and $1^{\text {th }}$ bus and $\mathrm{N}$ is the count of transmission lines.

$$
A=\sum_{i=1}^{N} g_{i}\left[\begin{array}{lll}
V_{k}^{2}+V_{l}^{2} & 2 V_{k} V_{l} \cos \left(\delta_{k}\right. & \left.\delta_{l}\right)
\end{array}\right]
$$

Minimization of voltage deviation: The formulation for the minimization of voltage deviation corresponds to the voltage magnitude $\mathrm{V}_{\mathrm{u}}$ corresponding to the reference voltage $\mathrm{V}_{\text {ref }}$ is represented in eq. (5) where LB denotes the count of load buses $\psi(x)$ represents the step function expressed in eq. (6).

$$
\begin{gathered}
B=\sum_{u=1}^{L B} P_{f} \psi\left(V_{\min } \quad V_{k}\right)+P_{f} \psi\left(V_{k} \quad V_{\max }\right) \\
1 ; \quad \text { if } \quad x \geq 0 \\
\psi(x)=0 ; \quad \text { otherwise }
\end{gathered}
$$

The formulation for computing the voltage $V_{k}$ is given in eq. (7) where

$$
\begin{aligned}
& \left|V_{k}\right|^{2}=\left|V_{u}\right|^{2} 2\left(\hat{w}_{u k} P_{u k}+\hat{f}_{u k} Q_{u k}\right)+c_{u k}(P, Q) \\
& \hat{w}_{u k}=\operatorname{Re}\left\{b b^{H}\right\} w_{u k}+\operatorname{Im}\left\{b b^{H}\right\} f_{u k} \\
& \hat{f}_{u k}=\operatorname{Re}\left\{b b^{H}\right\} \quad f_{u k} \quad \operatorname{Im}\left\{b b^{H}\right\} w_{u k} \\
& b=1 e^{j 2 \pi / 3} e^{j 2 \pi / 3} \\
& c_{u k}=\left[z_{u k}\left[S_{u k}^{*} . / V_{u}^{*}\right]\right] \quad\left[z_{u k}^{*}\left[S_{u k_{0}} \cdot / V_{u}\right]\right] \\
& z_{i m}=w+j f \\
& S_{u k}=\left[P_{u k}+j Q_{u k}\right]\left[\begin{array}{ll}
\hat{z}_{u k}\left(P_{u k}\right. & \left.j Q_{u k}\right)
\end{array}\right] \\
& \hat{z}_{u k}=\mathrm{z}_{\mathrm{uk}} \quad\left(b_{i} b_{i}^{H}\right)
\end{aligned}
$$

There is limit range of voltage that should be used by the power system which is called as the threshold voltage. Moreover, the power system should be fault tolerance. Voltage instability is one of the major problems arising in the power system, which is minimized to enhance the voltage stability index L index which is represented in eq. (15) where $1=1,2 \ldots \ldots ., \mathrm{NPQ}, \mathrm{NPV}$ is the sum of PV bus, $\mathrm{Y}_{\mathrm{A}}$ and $\mathrm{Y}_{\mathrm{B}}$ are the sub matrices.

$$
\begin{gathered}
L_{l}=\left|1 \sum_{k=1}^{N P V} F_{l k} \frac{V_{k}}{V_{l}}\right| \\
\mathrm{F}_{\mathrm{lk}}=\left[\mathrm{Y}_{\mathrm{A}}\right]^{-1}\left[\mathrm{Y}_{\mathrm{B}}\right]
\end{gathered}
$$

The formulation for the voltage stability is represented as,

$$
C=\max \left(L_{l}\right)
$$

\section{Satisfaction under equality and inequality constraints}


The formulation for the equality constraints can be represented in eq. (18) and eq. (19), where NB indicates the count of buses, the generation of the active and reactive power is denoted as $\mathrm{P}_{\mathrm{Gk}}$ and $\mathrm{Q}_{\mathrm{Gk}}$ of $\mathrm{k}^{\text {th }}$ bus, active and reactive power demand is denoted as $\mathrm{P}_{\mathrm{Dk}}$ and $\mathrm{Q}_{\mathrm{Dk}}$ of $\mathrm{k}^{\text {th }}$ bus, transfer conductance is denoted as $\mathrm{G}_{\mathrm{kl}}$ and suspectance is denoted as $\mathrm{B}_{\mathrm{kl}}$.

$$
\begin{array}{cccc}
P_{G k} & P_{D k} & V_{k} & \sum_{l=1}^{N B} V_{l}\left[\begin{array}{lll}
G_{k l} \cos \left(\delta_{k}\right. & \left.\delta_{l}\right)+B_{k l} \sin \left(\delta_{k}\right. & \left.\delta_{l}\right)
\end{array}\right]=0, \quad k=1,2 \ldots \ldots \ldots . . N B \\
Q_{G k} & Q_{D k} & V_{k} \sum_{l=1}^{N B} V_{l}\left[\begin{array}{lll}
G_{k l} \cos \left(\delta_{k}\right. & \left.\delta_{l}\right)+B_{k l} \sin \left(\delta_{k}\right. & \left.\delta_{l}\right)
\end{array}\right]=0, \quad l=1,2 \ldots \ldots \ldots . . N B
\end{array}
$$

The formulation of the equality constraints are expressed in following equations. The threshold limit of the output voltage magnitude and power of the generator is expressed as,

$$
\begin{gathered}
V_{G k}^{\min } \leq V_{G k} \leq V_{G k}^{\max }, k=1,2, \ldots \ldots . . N G \\
Q_{G k}^{\min } \leq Q_{G k} \leq Q_{G k}^{\max }, k=1,2, \ldots \ldots . . N G
\end{gathered}
$$

The resultant output of the shunt VAR compensators are represented as,

$$
Q_{C k}^{\min } \leq Q_{C k} \leq Q_{C k}^{\max }, k=1,2, \ldots \ldots . . N C
$$

The limit range of the transformer tap settings is represented as,

$$
T_{k}^{\min } \leq T_{k} \leq T_{k}^{\max }, k=1,2, \ldots \ldots . . N T
$$

The limit range of the magnitude of voltage at and the transmission line is represented as,

$$
\begin{array}{r}
V_{L k}^{\min } \leq V_{L k} \leq V_{L k}^{\max }, \mathrm{k}=1,2, \ldots \ldots . \mathrm{NPQ} \\
S_{l k} \leq S_{l k}^{\max }, k=1,2, \ldots \ldots . . N
\end{array}
$$

\section{ODFF based ORPD}

\subsection{Coventional Firefly}

Firefly algorithm [31] is one of the meta- heuristic algorithm that follows some basic rules such as, 1) All fireflies are unisexual 2) The attraction among diverse firefly is based on their brightness 3) The brightness of firefly is determined by the calculation of fitness function. The general formulation associated with the firefly algorithm is explained below.

Distance: The distance between the two fireflies $\mathrm{k}^{\text {th }}$ and $1^{\text {th }}$ is calculated by Cartesian distance that is represented in eq. (26) where $D$ denotes the number of dimensions.

$$
\left.d s_{k l}=\sqrt{\sum_{m=1}^{D}\left(v_{k, m}\right.} v_{l, m}\right)^{2}
$$

Attractiveness: The attractiveness of each firefly is expressed in eq. (27) where $\gamma$ denotes the absorption coefficient of light.

$$
\beta(\mathrm{ds})=\beta_{0} \mathrm{e}^{-\gamma \mathrm{ds} \mathrm{s}^{2}}
$$

Movement: The travelling of $\mathrm{k}^{\text {th }}$ firefly towards the more attractive $1^{\text {th }}$ is calculated using eq. (28) where $\alpha$ represents the randomization parameter, $\mathrm{v}_{\mathrm{k}}^{\text {new }}$ is the new position of $\mathrm{k}^{\text {th }}$ firefly and $\mathrm{v}_{\mathrm{k}}^{\text {old }}$ represents the old position of $\mathrm{k}^{\text {th }}$ firefly.

$$
v_{k}^{\text {new }}=v_{k}^{\text {old }}+\beta_{0} e^{\gamma d s^{2}}\left(v_{k}^{\text {old }} \quad v_{l}\right)+\alpha\left(\text { rand } \frac{1}{2}\right)
$$

The pseudo code of the conventional firefly is given below. 


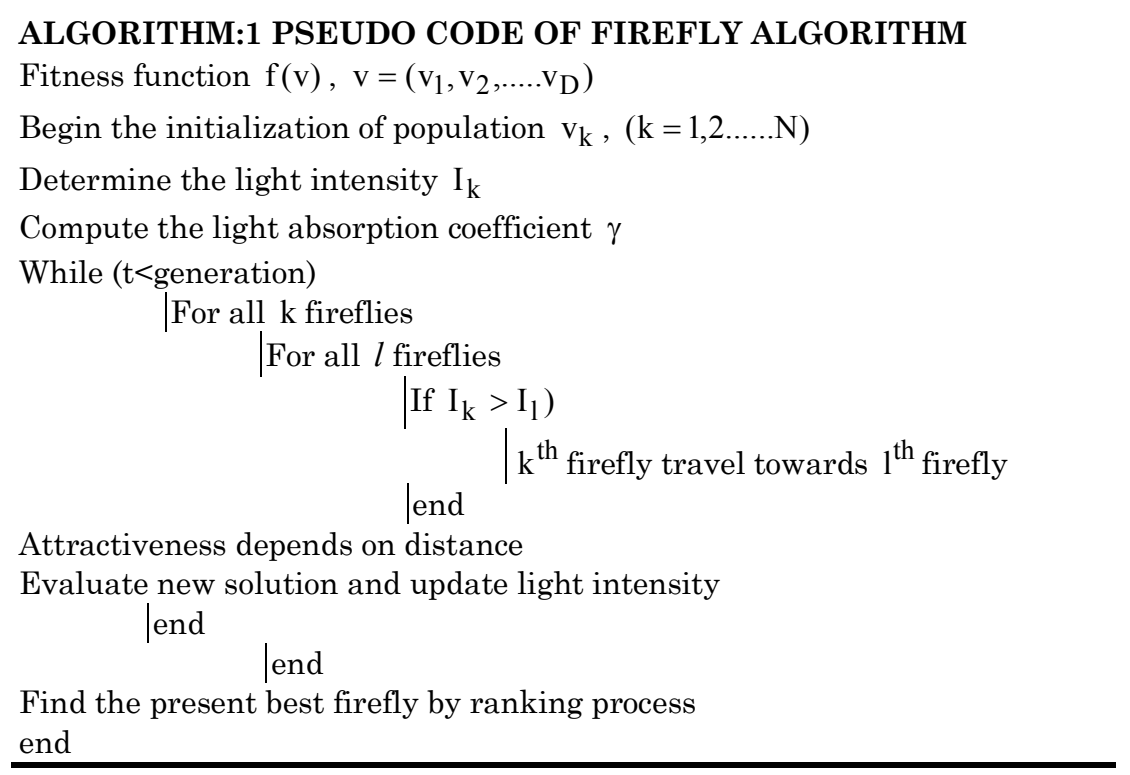

\subsection{Proposed ODFF Firefly}

The main limitation of normal firefly algorithm is that it does not consider all dimensions of a single firefly which leads to the problem of global optima. Instead, the proposed ODFF [32] precisely determines the global optima by the way of finding all the dimension of a single firefly. So here the best solution is the resultant that considers from all dimension of all fireflies and updating of position of all fireflies based on the global best firefly. The ODFF is used to overcome the two main challenges that arise in normal firefly 1) To enhance the convergence rate 2) To update the position of each firefly among diverse dimensions. The opposite position of the firefly is calculated using eq. (29) where, $\mathrm{k}=1,2 \ldots \mathrm{D}, 1=1,2 \ldots . \mathrm{N}$.

$$
\hat{v}_{k l}=i_{l}+j_{l}-v_{k l}
$$

Based on the global best firefly, the movement of $\mathrm{k}^{\text {th }}$ firefly towards the best firefly is represented as,

$$
\mathrm{v}_{\mathrm{k}}^{\text {new }}=\mathrm{v}_{\mathrm{k}}^{\text {old }}+\beta_{0} \mathrm{e}^{-\gamma \mathrm{ds}^{2}}\left(\mathrm{v}_{\mathrm{k}}^{\text {old }}-\text { Fbestpos }\right)+\alpha\left(\text { rand }-\frac{1}{2}\right)
$$

Here the opposite position of each firefly is computed. The initialization process of firefly in the proposed method is done efficiently, so that the convergence speed is increased and the computational complexity is reduced. The pseudo code of the proposed ODFF algorithm is depicted below.

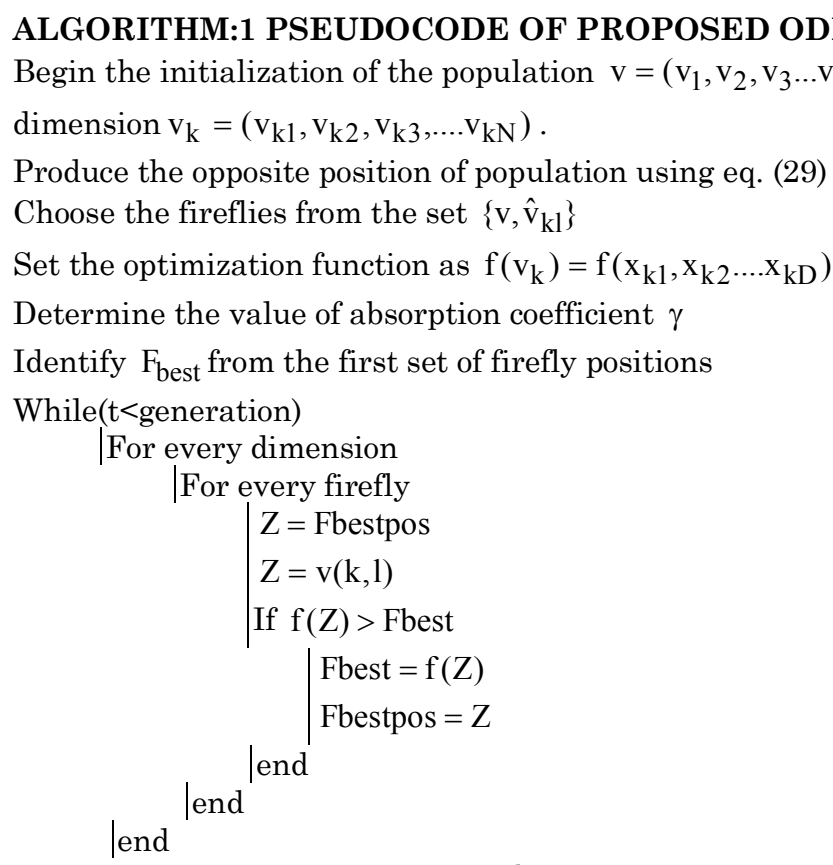

Find the present best firefly by ranking process end 
The flowchart of the aforementioned pseudo code is shown in fig. 1.

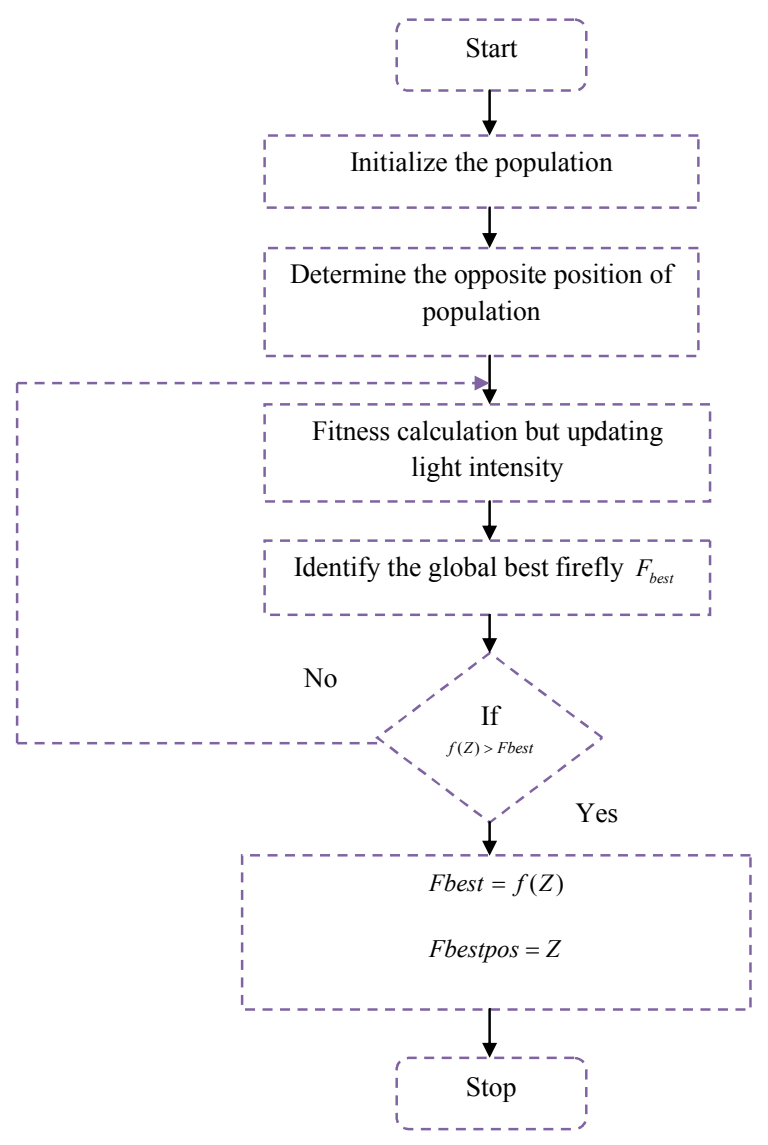

Fig. 1. Flowchart of ODFF approach

The description of the ODFF pseudo code and flowchart is explained below.

1 The initialization of the position of the firefly $\mathrm{v}$ is selected randomly.

2 Calculate the opposite position $\hat{v}$ of the set of firefly $v$ using eq. (29).

3 The optimization function the firefly is fixed and the absorption coefficient is also calculated.

4 Among the first set of fireflies, identify the global best firefly fbest. This best firefly is responsible for the initial population.

5 The update process is done according to the rule given in the pseudo code of the proposed ODFF algorithm. If the update firefly is greater than the global best firefly, then the global best firefly is replaced by the update firefly.

6 The new solutions are evaluated and the fitness function is calculated.

7 The best firefly is determined based on the ranking process.

8 The same procedure is repeated till; it has reached its maximum iteration.

\section{Results and Discussions}

\subsection{Experimental Setup}

The proposed ODFF algorithm was simulated in MATLAB under IEEE 14 and IEEE 39 standard test bus systems. The ORD problem was considered in this experiment and the objectives such as active power loss $A$ and the voltage deviation $B$ were determined. The performance of the proposed algorithm was analyzed by comparing it with other conventional algorithms like GA, PSO and ABC. The aforementioned algorithms are vastly dependant on initial solutions, so the result may not be optimum on a single run. So the experimentation was done for 5 times and the best and worst solution corresponding to their execution is determined. Further, the mean and median performance is examined from the best and worst solutions. Subsequently, the standard deviation is also determined to find the trustworthiness of the algorithm. 


\subsection{Performance Analysis of IEEE 14 Bus System}

The proposed ODFF algorithm is performed by fixing the control variables. The analysis of system without ORPD as well as with ORPD is shown in table 1. The control variables are fixed at five generator buses (bus. No. $1,2,3,6,8), 13^{\text {th }}$ and $3^{\text {rd }}$ voltage magnitude of bus and $8^{\text {th }}, 9^{\text {th }}$ and $10^{\text {th }}$ transformer tap settings of bus system.

Table 1. Analysis of system without ORPD and with ORPD of IEEE 14 test bus system

\begin{tabular}{lllllllllllll}
\hline Control variables & $\mathbf{Q , 1}$ & $\mathbf{Q , 2}$ & $\mathbf{Q , 3}$ & $\mathbf{Q , 6}$ & $\mathbf{Q , 8}$ & $\mathbf{V , 1 3}$ & $\mathbf{V , 3}$ & $\mathbf{T , 8}$ & $\mathrm{T}, \mathbf{9}$ & $\mathrm{T}, \mathbf{1 0}$ \\
\hline Without ORPD & & 0 & 12.7 & 19 & 7.5 & 0 & 1.05 & 1.01 & 0.98 & 0.98 & 0.93 \\
\hline \multirow{6}{*}{ With ORPD } & GA & 1.070 & 12.327 & 2.8097 & 17.172 & 2.8407 & 0.9906 & 0.90079 & 0.95 & 0.95 & 0.95 \\
& PSO & 8.862 & 4.650 & 8.004 & 8.165 & 14.842 & 0.9605 & 0.9846 & 0.94021 & 1.01 & 0.93552 \\
& FF & 3.703 & 13.08 & 7.987 & 6.6635 & 15.615 & 0.9862 & 1.0997 & 0.95 & 0.95 & 0.95 \\
& ABC & 8.577 & 2.957 & 3.7 & 2.033 & 8.845 & 0.928 & 0.947 & 1.0415 & 0.9769 & 0.9836 \\
& ODFF & 3.25 & 9.50 & 10.28 & 1.2 & 18.50 & 1.5 & 0.7 & 0.85 & 0.932 & 0.958 \\
\hline
\end{tabular}

The computation of active power loss and the voltage deviation of IEEE test bus system without ORPD and with ORPD of proposed ODFF compared with four conventional algorithms are shown in table 2. In the proposed ODFF method, the active power loss is $0.002 \%$ lower than system without ORPD, 0.001\% higher than GA, 0.002\% lower than PSO, 0.001\% higher than FF and 0.001\% higher than ABC.

Table 2. Active power loss and voltage deviation of system without OPRD and with ORPD of IEEE 14 test bus System

\begin{tabular}{lllll}
\hline \multicolumn{1}{l}{ Control variables } & & A & B & \multicolumn{1}{c}{ Final fitness } \\
\hline Without ORPD & & 13.393 & 1.4817 & 0.2974 \\
\hline With ORPD & GA & 13.364 & 1.4694 & 0.29496 \\
& PSO & 13.418 & 1.4868 & 0.29844 \\
& FF & 13.364 & 1.4694 & 0.29496 \\
& ABC & 13.368 & 1.5929 & 0.29496 \\
& ODFF & 13.39 & 1.461 & 0.2922 \\
\hline
\end{tabular}

The limited voltage used in the system should be 0.97 p.u to1.06 p.u. If the voltage is greater or lower than the threshold, the subsequent penalty should be added. Moreover, , the voltage deviation of the proposed ODFF method is $0.013 \%$ better than system without ORPD and $0.005 \%, 0.017 \%, 0.005 \%$ and $0.08 \%$ better than GA, PSO, FF and ABC algorithm. Even though, the active power loss and voltage deviation of the proposed method is higher than some of the conventional algorithm, the final fitness is less compared with conventional algorithms. The final fitness of ODFF is $0.017 \%, 0.009 \%, 0.20 \%, 0.009 \%$ and $0.017 \%$ better than the system without ORPD, GA, PSO, FF and ABC algorithms. The statistical report of proposed of ODFF algorithm with the conventional algorithms are shown in table 3 .

Table 3. Statistical report of IEEE 14 test bus system of proposed ODFF with conventional algorithms

\begin{tabular}{lllll|l}
\hline Metrics & Best & Worst & Mean & Median & $\begin{array}{l}\text { Standard } \\
\text { deviation }\end{array}$ \\
\hline GA & 0.29496 & 0.29496 & 0.29496 & 0.29496 & $6.674 \mathrm{e}-09$ \\
PSO & 0.296 & 0.29844 & 0.29694 & 0.29693 & 0.00093 \\
FF & 0.29496 & 0.29496 & 0.29496 & 0.29496 & $1.659 \mathrm{e}-10$ \\
ABC & 0.29496 & 0.29496 & 0.29496 & 0.29496 & $3.263 \mathrm{e}-09$ \\
ODFF & 0.2920 & 0.29785 & 0.2949 & 0.29432 & 0.00185 \\
\hline
\end{tabular}

The best case scenario is the occurrence of best convergence from five rounds where as the worst case scenario indicates the poor performance. The resultant output of the best case scenario of the proposed ODFF method is $0.010 \%, 0.13 \%, 0.010 \%$ and $0.010 \%$ better than GA, PSO, FF and ABC. Rather than in case of worst case scenario, the proposed method provides $0.001 \%$ better performance than PSO and $0.009 \%$ worst performance than GA, FF and ABC. However, the mean and median performance is better than the conventional methods. The mean performance of the proposed ODFF is $0.006 \%$ better than PSO and $0.002 \%$ better than GA, FF and ABC. The median performance is $0.0021 \%, 0.008 \%, 0.002 \%$, and $0.002 \%$ better than GA, PSO, FF and ABC. On the other hand, the standard deviation of the proposed method provides worst performance than the conventional methods, but the higher performance of mean and median overcomes the limitation in terms of deviation. 


\subsection{Performance Analysis of IEEE 39 Bus System}

The analysis of system without ORPD and with ORPD of IEEE 39 test bus system is shown in table 4 . To obtain the less power loss and voltage deviation, the reactive power of six generator bus (bus no. 31, 32, $35,38,33$ and 34 ) and transformer tap settings of $44^{\text {th }}, 38^{\text {th }}$ and $35^{\text {th }}$ buses are preset.

Table 4. Analysis of system without ORPD and with ORPD of IEEE 39 test bus system

\begin{tabular}{lllllllllllll}
\hline Control variables & $\mathbf{Q , 3 1}$ & $\mathbf{Q , 3 2}$ & $\mathbf{Q , 3 5}$ & $\mathbf{Q , 3 8}$ & $\mathbf{Q , 3 3}$ & $\mathbf{Q , 3 4}$ & $\mathrm{T}, 44$ & $\mathrm{~T}, 38$ & $\mathrm{~T}, 35$ & $\mathrm{~T}, 36$ \\
\hline Without ORPD & & 4.6 & 0 & 0 & 1.0265 & 0.9972 & 1.0123 & 1.025 & 1.07 & 1.006 & 1.006 \\
\hline & GA & 0.93619 & -0.69 & 0.3843 & 1.0753 & 1.088 & 1.0868 & 0.96451 & 1.05 & 1.0299 & 1.0418 \\
With ORPD & PSO & 0.33262 & -0.50 & -0.637 & 1.0484 & 0.97989 & 1.013 & 0.98367 & 1.0528 & 1.0135 & 1.0187 \\
& FF & 0.82819 & -0.084 & 0.85027 & 1.0259 & 1.0828 & 1.0522 & 0.96451 & 1.05 & 1.0299 & 1.0418 \\
& ABC & 0.13958 & 0.52142 & -0.6328 & 1.1 & 0.92709 & 1.045 & 0.96439 & 1.05 & 1.0093 & 1.0138 \\
& OBL-FF -0.97 & -0.48 & -0.752 & 1.080 & 1.087 & 0.895 & 0.9578 & 1.03 & 1.024 & 1.055 \\
\hline
\end{tabular}

The occurrence of active power loss and voltage deviation of the system without ORPD and with ORPD is shown in table 5. The active power loss of the proposed ODFF method provides $0.0011 \%$, $0.001 \%$, and $0.004 \%$ better than the system without ORPD and the system with ORPD that comprises GA, PSO and $0.008 \%$ and $0.023 \%$ worst performance than FF and ABC. Further, the voltage deviation of the proposed ODFF is $0.140 \%$ better than the system without ORPD. Then the proposed method is $0.006 \%, 0.219 \%$ and $0.28 \%$ better than GA, PSO and FF, but it generates $0.0379 \%$ worst performance than ABC algorithm. Even though, the active power loss and voltage deviation of the proposed method is worst in some cases, the final fitness shows it superiority than the conventional methods. The final fitness of the proposed ODFF method is $0.070 \%, 0.001 \%, 0.006 \%, 0.005 \%$ and $0.002 \%$ better than GA, $\mathrm{PSO}, \mathrm{FF}$ and ABC.

Table 5. Active power loss and voltage deviation of system without OPRD and with ORPD of IEEE 39 test bus system

\begin{tabular}{lllll}
\hline Control variables & & A & B & Final fitness \\
\hline Without ORPD & & 43.591 & 50.352 & 38.99 \\
\hline With ORPD & GA & 43.147 & 8.9004 & 36.298 \\
& PSO & 42.755 & 11.325 & 36.469 \\
& FF & 43.637 & 12.334 & 36.304 \\
& ABC & 43.28 & 8.5168 & 36.327 \\
& ODFF & 43.10 & 8.84 & 36.248 \\
\hline
\end{tabular}

The statistical report of IEEE 39 bus system of proposed ODFF method with conventional methods is shown in table 6 . The best case scenario of the proposed method is $0.005 \%, 0.0008 \%, 0.005 \%$ and $0.005 \%$ better than GA, PSO, FF and ABC approaches. Subsequently, the worst case scenario of the proposed method is $0.007 \%, 0.005 \%, 0.009 \%$ and $0.005 \%$ better than GA, PSO, FF and ABC. Collectively, the mean and median performance of the performance of the proposed method is $0.909 \%, 0.002 \%$ better than the conventional algorithms.

Table 6. Statistical report of IEEE 39 test bus system of proposed ODFF with conventional algorithms

\begin{tabular}{llllll}
\hline Metrics & Best & Worst & Mean & Median & Standard deviation \\
\hline GA & 36.298 & 36.305 & 36.301 & 36.298 & 0.00359 \\
PSO & 36.28 & 36.491 & 36.413 & 36.469 & 0.09631 \\
FF & 36.298 & 36.313 & 36.303 & 36.302 & 0.00630 \\
ABC & 36.298 & 36.482 & 36.359 & 36.327 & 0.0775 \\
ODFF & 36.277 & 36.277 & 3.285 & 36.38 & 0.00058 \\
\hline
\end{tabular}

\section{Conclusion}

A method of reducing the active power loss and voltage deviation in ORPD system was presented in this paper. An improved method of firefly called ODFF was used and the corresponding performance was carried out in IEEE 14 and IEEE 39 test bus systems. This method has used the opposition based learning which leads to increase the convergence speed. The performance analysis of the proposed OFF method was compared with the conventional methods like GA, PSO, FF and ABC. Moreover, the statistical report associated with the performance of mean, median and standard deviation was also determined and was analyzed. Hence, the proposed ODFF methods have provided its superiority among the conventional approaches. 


\section{Compliance with Ethical Standards}

Conflicts of interest: Authors declared that they have no conflict of interest.

Human participants: The conducted research follows the ethical standards and the authors ensured that they have not conducted any studies with human participants or animals.

\section{References}

[1] B. A. Robbins and A. D. Dominguez-Garcia, "Optimal Reactive Power Dispatch for Voltage Regulation in Unbalanced Distribution Systems," in IEEE Transactions on Power Systems, vol. 31, no. 4, pp. 2903-2913, July 2016.

[2] Mehdi Mehdinejad, Behnam Mohammadi-Ivatloo, Reza Dadashzadeh-Bonab and Kazem Zare, "Solution of optimal reactive power dispatch of power systems using hybrid particle swarm optimization and imperialist competitive algorithms", International Journal of Electrical Power \& Energy Systems, vol. 83, pp.104-116, December 2016.

[3] Seyed Masoud Mohseni-Bonab, Abbas Rabiee and Behnam Mohammadi-Ivatloo, "Voltage stability constrained multi-objective optimal reactive power dispatch under load and wind power uncertainties: A stochastic approach", Renewable Energy, vol. 85, pp.598-609, 2016.

[4] A. Mukherjee and V. Mukherjee, "Solution of optimal reactive power dispatch by chaotic krill herd algorithm," in IET Generation, Transmission \& Distribution, vol. 9, no. 15, pp. 2351-2362, 2015.

[5] M. Basu, "Multi-objective optimal reactive power dispatch using multi-objective differential evolution", Electrical Power and Energy Systems, vol.82, pp.213-224, 2016.

[6] Mohd Herwan Sulaimana, Zuriani Mustaffab, Mohd Rusllim Mohamed and Omar Alimanaa, "Applied Soft Computing, vol. 32, pp.286-292, 2015.

[7] K. Aoki, M. Fan and A. Nishikori, "Optimal VAr planning by approximation method for recursive mixed-integer linear programming," in IEEE Transactions on Power Systems, vol. 3, no. 4, pp. 1741-1747, Nov 1988.

[8] S. S. Sachdeva and R. Billinton, "Optimum Network Var Planning by Nonlinear Programming," in IEEE Transactions on Power Apparatus and Systems, vol. PAS-92, no. 4, pp. 1217-1225, July 1973.

[9] M. Bjelogrlic, M. S. Calovic, P. Ristanovic and B. S. Babic, "Application of Newton's optimal power flow in voltage/reactive power control," in IEEE Transactions on Power Systems, vol. 5, no. 4, pp. 1447-1454, Nov 1990.

[10] R. Mota-Palomino and V. H. Quintana, "Sparse Reactive Power Scheduling by a Penalty Function - Linear Programming Technique," in IEEE Transactions on Power Systems, vol. 1, no. 3, pp. 31-39, Aug. 1986.

[11] F. C. Lu and Y. Y. Hsu, "Reactive power/voltage control in a distribution substation using dynamic programming," in IEE Proceedings - Generation, Transmission and Distribution, vol. 142, no. 6, pp. 639-645, Nov 1995.

[12] N. Deeb and S. M. Shahidehpour, "Linear reactive power optimization in a large power network using the decomposition approach," in IEEE Transactions on Power Systems, vol. 5, no. 2, pp. 428-438, May 1990.

[13] V. H. Quintana and M. Santos-Nieto, "Reactive-power dispatch by successive quadratic programming," in IEEE Transactions on Energy Conversion, vol. 4, no. 3, pp. 425-435, Sep 1989.

[14] Wu QH, Cao YJ, Wen JY, "Optimal reactive power dispatch using an adaptive genetic algorithm", Electr Power Energy Syst, vol.20, no.8, pp.563-569, 1998.

[15] S. Mishra, "Bacteria foraging based solution to optimize both real power loss and voltage stability limit," Power Engineering Society General Meeting, 2007. IEEE, Tampa,pp. 1-1, 2007.

[16] H. Yoshida, K. Kawata, Y. Fukuyama, S. Takayama and Y. Nakanishi, "A particle swarm optimization for reactive power and voltage control considering voltage security assessment," in IEEE Transactions on Power Systems, vol. 15, no. 4, pp. 1232-1239, Nov 2000.

[17] J. G. Vlachogiannis and K. Y. Lee, "Quantum-Inspired Evolutionary Algorithm for Real and Reactive Power Dispatch," in IEEE Transactions on Power Systems, vol. 23, no. 4, pp. 1627-1636, Nov. 2008.

[18] A. A. A. Esmin, G. Lambert-Torres and A. C. Zambroni de Souza, "A hybrid particle swarm optimization applied to loss power minimization," in IEEE Transactions on Power Systems, vol. 20, no. 2, pp. 859-866, May 2005.

[19] Q. H. Wu and J. T. Ma, "Power system optimal reactive power dispatch using evolutionary programming," in IEEE Transactions on Power Systems, vol. 10, no. 3, pp. 1243-1249, Aug 1995.

[20] Ruey-Hsun Liang, Jia-Ching Wang, Yie-Tone Chen, Wan-Tsun Tseng, " An enhanced firefly algorithm to multiobjective optimal active/reactive power dispatch with uncertainties consideration," Electrical Power and Energy Systems, vol. 64, pp. 1088 - 1097, 2015.

[21] [21] M. Basu, " Quasi-oppositional differential evolution for optimal reactive power dispatch," Electrical Power and Energy Systems, vol. 78, pp. 29-40, 2016.

[22] Ali Ghasemia, Khalil Valipourb and Akbar Tohidic, "Multi objective optimal reactive power dispatch using a new multi objective strategy", International Journal of Electrical Power \& Energy Systems, vol.57, pp. 318-334, May 2014.

[23] Amit Saraswat and Ashish Saini, "Multi-objective optimal reactive power dispatch considering voltage stability in power systems using HFMOEA", Engineering Applications of Artificial Intelligence, vol. 26, no. 1, pp.390404, January 2013. 
[24] R. Storn, K. Price, "Minimizing the real functions of the ICEC'96 contest by differential evolution," Evolutionary Computation, pp. 842-844, 1996.

[25] Kanagasabai Lenin, Bhumanapally Ravindhranath Reddy AND Munagala Suryakalavathi, "Hybrid Tabu search-simulated annealing method to solve optimal reactive power problem", International Journal of Electrical Power \& Energy Systems, vol. 82, pp.87-91, November 2016.

[26] Susanta Dutta, Pranabesh Mukhopadhyay, Provas Kumar Roy and Debashis Nandi, "Unified power flow controller based reactive power dispatch using oppositional krill herd algorithm", International Journal of Electrical Power \& Energy Systems, vol.80, pp. 10-25, September 2016.

[27] Barun Mandal and Provas Kumar Roy, "Optimal reactive power dispatch using quasi-oppositional teaching learning based optimization", International Journal of Electrical Power \& Energy Systems, vol.53, pp. 123-134, 2013.

[28] Rudra Pratap Singh, V. Mukherjee and S.P. Ghoshal, "Optimal reactive power dispatch by particle swarm optimization with an aging leader and challengers", Applied Soft Computing, vol. 29, pp. 298-309, April 2015.

[29] Abhishek Rajan and T. Malakar, "Optimal reactive power dispatch using hybrid Nelder-Mead simplex based firefly algorithm", International Journal of Electrical Power \& Energy Systems, vol., pp.9-24, March 2015.

[30] Binod Shaw, V. Mukherjee and S.P. Ghoshal, "Solution of reactive power dispatch of power systems by an opposition-based gravitational search algorithm", International Journal of Electrical Power \& Energy Systems, vol.55, pp.29-40, February 2014.

[31] Amir Hossein Gandomi, Xin-She Yang and Amir Hossein Alavi, "Mixed variable structural optimization using Firefly Algorithm", Computers \& Structures, vol.89, no.23-24, pp. 2325-2336, December 2011.

[32] Om Prakash Verma, Deepti Aggarwal and Tejna Patodi, "Opposition and dimensional based modified firefly algorithm", Expert Systems with Applications, vol. 44, pp. 168-176, February 2016. 